\title{
New approaches for improving the production of the 1st and 2nd generation ethanol by yeast*
}

\author{
Olena Kurylenko1, Marta Semkiv1, Justyna Ruchala2, Orest Hryniv', Barbara Kshanovska1, \\ Charles Abbas ${ }^{3}$, Kostyantyn Dmytruk ${ }^{1}$ and Andriy Sibirny ${ }^{1,2 区}$ \\ IInstitute of Cell Biology, NAS of Ukraine, Lviv, Ukraine; ${ }^{2}$ Rzeszow University, Rzeszow Poland; ${ }^{3}$ ADM Research, N. Brush, Decatur IL, USA
}

Increase in the production of 1st generation ethanol from glucose is possible by the reduction in the production of ethanol co-products, especially biomass. We have developed a method to reduce biomass accumulation of Saccharomyces cerevisiae by the manipulation of the intracellular ATP level due to overexpression of genes of alkaline phosphatase, apyrase or enzymes involved in futile cycles. The strains constructed accumulated up to $10 \%$ more ethanol on a cornmeal hydrolysate medium. Similar increase in ethanol accumulation was observed in the mutants resistant to the toxic inhibitors of glycolysis like 3-bromopyruvate and others. Substantial increase in fuel ethanol production will be obtained by the development of new strains of yeasts that ferment sugars of the abundant lignocellulosic feedstocks, especially $x y-$ lose, a pentose sugar. We have found that xylose can be fermented under elevated temperatures by the thermotolerant yeast, Hansenula polymorpha. We combined protein engineering of the gene coding for xylose reductase (XYL1) along with overexpression of the other two genes responsible for xylose metabolism in yeast (XYL2, XYL3) and the deletion of the global transcriptional activator CAT8, with the selection of mutants defective in utilizing ethanol as a carbon source using the anticancer drug, 3-bromopyruvate. Resulted strains accumulated 20-25 times more ethanol from xylose at the elevated temperature of $45^{\circ} \mathrm{C}$ with up to $12.5 \mathrm{~g} \mathrm{~L}^{-1}$ produced. Increase in ethanol yield and productivity from xylose was also achieved by overexpression of genes coding for the peroxisomal enzymes: transketolase (DAS1) and transaldolase (TAL2), and deletion of the ATG13 gene.

Key words: Saccharomyces cerevisiae, metabolic engineering, classical selection, xylose, Hansenula polymorpha

Received: 31 July, 2015; revised: 08 September, 2015; accepted: 27 October, 2015; available on-line: 30 November, 2015

\section{INTRODUCTION}

Alcoholic fermentation dates back at least 6000 years B.C. Until recently, the major discoveries in this field were improvements in distillation and the isolation and identification of new yeast responsible for fermentation which enabled their use in pure culture. In the past, ethanol yeast strain improvement methods relied heavily on classical genetic breeding techniques that involved mating and hybridization, as well as classical mutagenesis. Most recently, new approaches that rely on genetic engineering in combination with evolutionary adaptation and rational design, have gained greater acceptance.
Today, the industrial scale production of fuel ethanol uses conventional feedstocks such as glucose (derived from starch) and sucrose, and is known as 1st generation (1G). In 2014, about 93 billion liters of such ethanol were produced (http://www.afdc.energy.gov/data/10331). The production of 1 st generation ethanol represents the largest industrial biotechnological application of yeast. For this reason, new scientific approaches to improve yeast strains have to be developed to increase yield and productivity to reduce cost of commercial production. Ethanol is produced from glucose or sucrose by Saccharomyces cerevisiae under anaerobic conditions as one of the end products of sugar catabolism. The pathway for the production of ethanol from glucose in yeast (known as glycolysis or Embden-Meyerhof-Parnas, EMP, pathway), yields 2 moles of ATP from one mole of glucose. The ATP produced is subsequently used to drive the conversion of the products derived from glucose to produce cellular biomass. When yeast are cultivated under anaerobic conditions, $93 \%$ of the glucose used is converted to ethanol with the remaining $7 \%$ primarily being converted into cell biomass. In addition to ethanol, there are other low-molecular weight compounds produced, among which glycerol is the most prominent one (nearly $4 \%$ of that of ethanol). Theoretically, it is possible to increase ethanol yield and productivity by decreasing the accumulation of cellular biomass and glycerol. As cellular biomass is the largest byproduct of ethanol production, it represents an area of great research interest. There are several metabolic engineering approaches to increase ethanol yield by reducing glycerol accumulation in yeast (Gombert \& van Maris, 2015). Briefly speaking, $\mathrm{NADH}$ accumulated in yeast during biomass production, can be used for the reduction of acetate and/or to decrease the enzymes involved in glycerol synthesis from dihyroxyacetone-3-phosphate. By comparison to glycerol reduction, much less is known on ways to achieve an increase in ethanol yield by decreasing biomass production. There have been several attempts to reduce ATP production by the engineering of yeast via the substitution of EMP pathway genes with Zymomonas mobilis genes for the Entner-Doudoroff (ED) pathway which yields only 1 mole of ATP per mole of glucose (Doelle, 1975). The ED pathway is used during alcoholic fermentation by the bacterium Zymomonas mobilis (Panesar et al., 2006) to produce ethanol from glucose and sucrose. As a result, Z. mobilis converts $97 \%$ of glucose to ethanol

e-mail: sibirny@cellbiol.Iviv.ua

*The results were presented at the 6 th International Weigl Conference on Microbiology, Gdańsk, Poland (8-10 July, 2015).

Abbreviations: 
with only $3 \%$ of the sugar converted to cell biomass. Attempts to express of $Z$. mobilis genes coding for a specific aldolase and dehydratase in $S$. cerevisiae were only partially successful, as transformants did not demonstrate activity of dehydratase, an iron-sulfur protein (Benisch \& Boles, 2014). Other approaches used were based on the activation of enzymes involved in ATP degradation, such as acid phosphatase Pho5 (Rogers \& Szostak, 1993), $F_{0}$, subunit of the membrane ATPase (Jensen et al., 2006), and SSB1 part of a chaperon exhibiting ATPase activity (Dmytruk et al., 2012). The corresponding results are available only in patent literature. We have found recently, that the overexpression of the vacuolar alkaline phosphatase Pho8 leads to an increase in yield and productivity of ethanol synthesis from glucose in laboratory and industrial strains of $S$. cerevisiae, whereas expression of the truncated, cytosol localized form, is detrimental to the cells (Semkiv et al., 2014). However, other approaches for controlling the decrease in ATP content in the cells and corresponding increase in ethanol production have to be investigated. It is also important to develop reliable methods for positive selection of ethanol overproducing strains.

The future of bioethanol industry belongs to 2nd generation ethanol that is produced from lignocellulose (Hahn-Hagerdal et al., 2006). However, lignocellulose is a very complex feedstock consisting of several types of polymers that are made up primarily of glucose and xylose. Xylose is the second most abundant sugar in nature but it is never present as a free monosaccharide. For this reason, it is thought that there are a few microorganisms that metabolize xylose and even fewer that can ferment this monosaccharide to ethanol with good yield and productivity. To be commercially viable, it is very important to combine the alcoholic fermentation of lignocellulosic sugars with enzymatic hydrolysis of cellulose and hemicellulose, using the process that is known as simultaneous saccharification and fermentation or SSF (Olofsson et al., 2008). For this, the fermenting organisms have to tolerate elevated temperatures, preferably close to $50^{\circ} \mathrm{C}$. We have described in detail the development of the thermotolerant Hansenula polymorpha yeast that are capable of alcoholic fermentation of glucose, cellobiose and xylose, however, ethanol yield and productivity from xylose was very low (Ryabova et al., 2003). During the last decade, properties of xylose alcoholic fermentation in $H$. polymorpha were further significantly improved by using methods of metabolic engineering and classical selection, however, ethanol yield and productivity are not as of today commercially feasible. In this paper we present a short review of our recent data on improvement of ethanol producers of the 1 st $(S$. cerevisiae $)$ and 2 nd (H. polymorpha) generations.

\section{IMPROVEMENT OF S. CEREVISIAE STRAINS FOR 1st GENERATION ETHANOL PRODUCTION}

S. cerevisiae is characterized by desirable industrial properties which include fast growth, efficient glucose anaerobic metabolism, high ethanol productivity, high yield, and tolerance to several environmental stress-factors, such as high ethanol concentration and low oxygen level (Piškur \& Langkjaer, 2004). Nevertheless, the ethanol yields from glucose and sucrose can be further increased. There are several approaches that can be employed for this and these consist of the use of genetic engineering techniques that target the redirection of yeast metabolism towards increased yield of ethanol production from carbohydrates by reducing co-product generation. Particularly, this can be achieved through the reduction of biomass synthesis, since this represents one of the major by-products during bioethanol production.

Under anaerobic conditions, alcoholic fermentation of sugars is the primary pathway in yeast that provides energy in the form of ATP for cellular maintenance and, if sufficient ATP is available, for continued growth that is necessary to produce ethanol. When ATP is used for growth, yeast biomass is formed at the expense of feedstock that is not converted to ethanol. Consequently, the reduction in yeast biomass production is expected to result in increased ethanol yields (Gombert \& van Maris, 2015). Here, we present several approaches leading to intracellular ATP exhaustion. These consist of the overexpression of ATP-degrading alkaline phosphatase, apyrase, and the generation of futile cycles by simultaneous expression of genes coding for phosphofructokinase and fructose-1,6-biphosphatase or the genes coding for pyruvate carboxylase and phosphoenolpyruvate carboxykinase.

\section{Alkaline phosphatase}

The $S$. cerevisiae nonspecific alkaline phosphatase (phosphomonoesterase) is located in the vacuole and encoded by the PHO8 gene. This enzyme catalyzes the dephosphorylation of many phosphate containing compounds that include ATP (Kaneko et al., 1982; Klionsky et al., 1989). Therefore, this enzyme may operate as nonspecific ATPase.

Intact and truncated versions of the $S$. cerevisiae PHO8 gene coding for vacuolar or cytosolic forms of alkaline phosphatase were fused with the alcohol dehydrogenase gene $(A D H 1)$ promoter in constructed plasmid for multicopy integration. Laboratory and industrial ethanol producing strains BY4742 and AS400 overexpressing vacuolar form of alkaline phosphatase were characterized by a slightly lowered intracellular ATP level with reduced biomass accumulation and by an increase in ethanol production $(13 \%$ and $7 \%)$ when compared to the parental strains (Semkiv et al., 2014). The results obtained can be explained by the accelerated hydrolysis of substrate due to the increased activity from the overexpression of vacuolar alkaline phosphatase. The target substrates are polyphosphates which are known to be the natural substrates of alkaline phosphatases (Fernandez et al., 1981). As the synthesis of polyphosphates requires ATP, an increase in alkaline phosphatase activity may lead to a futile cycle of ATP consumption that is necessary to replenish again the vacuolar polyphosphate pool (Klionsky et al., 1990). In contrast, strains of yeast that are engineered to express the truncated cytosolic form of alkaline phosphatase, showed a prolonged lag-phase, reduced biomass accumulation and a strong defect in ethanol production (Semkiv et al., 2014). For this reason, it is hypothesized that the cytosolic form of alkaline phosphatase decreases not only the level of ATP, but also that of other sugar phosphate intermediates, as well as regulators like fructose-2,6-bisphosphate, thereby leading to detrimental effect on ethanol production. However, confirmation of this hypothesis requires additional experiments.

\section{Apyrase}

ATP-diphosphohydrolases (apyrases) (EC 3.6.1.5), are enzymes hydrolyzing both the $\gamma$ - and $\beta$-phosphates of ATP and ADP. They are distinct from other phosphohydrolases with respect to their specific activity, nucleotide substrate specificity, divalent cation requirement, 
and sensitivity to inhibitors (Plesner, 1995; Handa et al., 1996). Apyrases are ubiquitously expressed in eukaryotes and have additionally been found in some prokaryotes, indicating a general role for these enzymes across species. In our experiments, bacterial apyrase apy from Escherichia coli was used for decreasing the yeast intracellular ATP level.

An apyrase gene, apy from E. coli lacking N-terminal periplasmic targeting sequence was functionally expressed in $S$. cerevisiae under the control of galactose-inducible GAL1 promoter. The corresponding representative transformant possessed a slight growth retardation, while the ATPase activity was increased by $21 \%$, as compared to the control strain containing empty plasmid. The ethanol accumulation and yield of the constructed strain revealed $17 \%$ and $28 \%$ increase, as compared to the control, during fermentation of a galactose containing media $(1 \%$ glucose $+10 \%$ galactose) (Dmytruk et al., 2012).

\section{Futile cycles}

Fructose-1,6-biphosphatase (FBPase) (EC 3.1.3.11) is one of the major gluconeogenesis enzymes, which hydrolyze D-fructose-1,6-biphosphate to D-fructose-6-phosphate in an ATP-dependent reaction. Simultaneous action of two enzymes - phosphofructokinase and fructose-1,6-biphosphatase - leads to futile cycle generation between D-fructose-1,6-biphosphate and D-fructose-6-phosphate leading to ATP dissipation. In living cells, there are multiple regulatory mechanisms of the FBP gene and enzyme activity, such as catabolic repression, inactivation through ubiquitination, inhibition by AMP and fructose-2,6-biphosphate (Navas \& Gancedo, 1996). Therefore, intracellular enzyme activity maintains basal level on media containing fermentable carbon sources.

To overcome such drawbacks of yeast FBPase, the bacterial FBPase insensitive to fructose-2,6-biphosphate inhibition was expressed in yeast (Navas \& Gancedo, 1996). In an effort to reproduce that data, we expressed FBPase from E. coli under control of a constitutive promoter of the TPI1 gene, encoding triose phosphate isomerase, in $S$. cerevisiae BY4742 strain in frame of a replicative plasmid.

Fermentation with two independent transformants was performed on YPD medium. Strains BY4742/fbp1_7 and BY4742/fbp1_13 synthesized 23.3 and $24.5 \mathrm{~g} \mathrm{~L}^{-1}$ of ethanol, respectively, while control strain harboring basal plasmid pRS42H (Taxis \& Knop, 2006) synthesized only $21.3 \mathrm{~g} \mathrm{~L}^{-1}$ (unpublished data). After re-calculation of obtained data, taking into account the production of yeast biomass, an increase of 5.2 and $8.8 \%$ in ethanol yield was reached (unpublished data). The ATP level of these strains was also measured. Total ATP level of analyzed transformants was decreased by $31-39 \%$, as compared to the control strain (unpublished data).

Specific activity of fructose-1,6-bisphosphatase of these strains was measured after 1 day of cultivation on synthetic medium supplemented with $2 \%$ glucose and compared with FBPase activity of a wild type strain BY4742, grown on glucose or ethanol as a sole carbon source. Specific FBPase activity of the obtained recombinant strains was 1.5-3-folds higher than that of the initial BY4742 strain. However, FBPase activity is still around 2-folds lower than that of the wild-type strain (WT) cultivated on ethanol containing medium as a sole carbon source (glyconeogenetic substrates derepress FBP1 gene expression in yeast). Further improvement of $f b p 1 E c$ gene expression is required. Obtained results are in agreement with previously published data (Navas \& Gancedo, 1996).

Another way to initiate the futile cycles is via the simultaneous activation of the pyruvate carboxylase and phosphoenolpyruvate carboxykinase enzymes. Pyruvate carboxylase uses ATP energy to convert pyruvate into oxaloacetate, and phosphoenolpyruvate carboxykinase uses ATP energy to convert oxaloacetate into phosphoenolpyruvate. At the same time, ATP is synthesized when phosphoenolpyruvate is converted into pyruvate by pyruvate kinase. Therefore, the resulting total loss in ATP is one molecule for one turn of the cycle.

In order to increase pyruvate carboxylase activity, the promoter of PYC2 gene was substituted by the strong constitutive TEF1 promoter. The selected recombinant strain showed a 3-5-fold increase in the specific activity of target enzyme, when compared to the initial strain.

As S. cerevisiae phosphoenolpyruvate carboxykinase activity is carefully regulated at the post-translational lev$\mathrm{el}$, and in order to avoid regulation, it was decided to use the gene coding for the corresponding enzyme from $E$. coli. The expression cassette containing the E. coli pckA gene coding for phosphoenolpyruvate carboxykinase under the control of $A D H 1$ gene promoter was integrated into the genome of the previously isolated strain overexpressing PYC2 gene. The specific pyruvate carboxylase and phosphoenolpyruvate carboxykinase activities were elevated by 3-4 and 6-7-folds, as compared to parental BY4742 strain, respectively. Ethanol production by constructed recombinant strains revealed a 2 -fold increase when compared to initial strain (unpublished data).

\section{Classical selection}

An alternative approach to increase ethanol production that relied on classical mutagenesis and selection, was used to isolate mutants within a population with improved fermentation performance (Chambers et al., 2009).

The method we describe here relied on the positive selection of industrial $S$. cerevisiae strains with enhanced ethanol production. The selective agents: oxythiamine, trehalose, 3-bromopyruvate, glyoxylic acid and glucosamine, were selected for their inhibitory effect on the enzymes involved in alcoholic fermentation and stress response.

Oxythiamine is a thiamine antagonist that, after its conversion to oxythiamine pyrophosphate, can bind to the active centers of thiamine-dependent enzymes. In particular, the specific activities of transketolase and pyruvate decarboxylase are affected by oxythiamine (Tylicki et al., 2005). It was speculated that yeast strains resistant to oxythiamine will have an increase in pyruvate to acetaldehyde conversion that can result in the increased production of ethanol. During the alcoholic fermentation, yeast cells are subjected to stress that could affect their viability and fermentation efficiency. It is known that trehalose functions as one of the major stress protectants and that the synthesis of trehalose is induced by several documented stress conditions at the transcriptional level in S. cerevisiae (Kaino \& Takagi, 2008). Trehalose-6-phosphate synthase is the key enzyme involved in the synthesis of trehalose and this enzyme is known to be inhibited by high concentrations of trehalose (Lippert et al., 1993). Yeast mutants resistant to increased concentrations of trehalose may have elevated intracellular concentration of this disaccharide, with subsequent beneficial effect of trehalose on ethanol production. The bro- 
mopyruvate (3-bromopyruvic acid) compound is known to be an efficient inhibitor of hexokinase and has been shown to cause severe depletion of cellular ATP (Ko et al., 2001; Geschwind et al., 2004). This leads to the disruption of glycolysis. Yeast mutants resistant to bromopyruvate may exhibit an increased level of glycolysis with elevated amount of synthesized ethanol. Glyoxylic acid is a known irreversible inhibitor of pyruvate decarboxylase activity (Uhlemann \& Schellenberger, 1976), the key enzyme in ethanol synthesis. Therefore, mutants resistant to glyoxylic acid may have an improved characteristic of alcoholic fermentation. Glucosamine (2-deoxy-2-aminoglucose) is a structural analog of glucose. Due to the absence of hydroxyl at the second position, this compound cannot be isomerized to the ketohexose (analog of fructose) by the glycolytic enzyme phosphoglucoisomerase Pgi1 (Bekesi et al., 1969). This leads to the disruption of the glycolysis at this step. The glucosamine resistant mutants could have a derepression of PGI1 or the entire regulon involved in regulation of the majority of glycolytic enzymes, leading to an increase in glucose flux through the glycolysis pathway.

We were able to obtain a set of mutants resistant to toxic concentrations of the selective agents: oxythiamine, trehalose, 3-bromopyruvate, glyoxylic acid and glucosamine, on the bases of industrial ethanol producing strain of $S$. cerevisiae AS400. Corresponding mutants had an increase in ethanol production by $7 \%, 5 \%, 6 \%$, $8 \%$ and $6 \%$ (Dmytruk et al., in press). In addition, and by using the combination of glyoxylic acid and glucosamine or glyoxylic acid, glucosamine and bromopyruvate in two- or three-step selection schemes, led to 10 or $12 \%$ increase in ethanol production over that obtained from the parental strain (Dmytruk et al., in press). The reasons for the increased ethanol production by the isolated mutants still remain unknown. We suggest that the observed increase in ethanol accumulation is caused by a decrease in the accumulation of fermentation by-products, e.g. biomass, glycerol or acetate. The identification of the mutated genes in the selected mutants is the subject of our current work. The results we obtained so far allow us to propose that oxythiamine, trehalose, bromopyruvate, glyoxylic acid and glucosamine are very efficient agents for the selection of yeast strains with improved efficacy of alcoholic fermentation. Moreover, the combination of glyoxylic acid and glucosamine or glyoxylic acid, glucosamine and bromopyruvate, when used in two- or three-step selection schemes, can result in an elevation of ethanol production over one-step selection. Isolated strains can be used for industrial ethanol production (Dmytruk et al., in press).

\section{IMPROVEMENT EFFICIENCY OF HIGH TEMPERATURE XYLOSE ALCOHOLIC FERMENTATION BY H. POLYMORPHA}

The thermotolerant methylotrophic yeast Hansenula polymorpha is capable of alcoholic fermentation of xylose at elevated temperatures $\left(45-48^{\circ} \mathrm{C}\right)$ (Ryabova et al., 2003; Ishchuk et al., 2009). Such characteristic of this yeast suggests it to be a good candidate for use in efficient process of simultaneous saccharification and fermentation (SSF). This process combines enzymatic hydrolysis of lignocellulosic material with subsequent fermentation of sugars in the same vessel at high temperatures, since temperature optima for cellulases and hemicellulases lay within 50 and $60^{\circ} \mathrm{C}$ (Olofsson et al., 2008). In our laboratory, several successful attempts were made to im- prove further this yeast's thermotolerance (Ishchuk et al., 2009). At an industrial scale, increased thermotolerance can reduce cooling costs and the risk of contamination with more energy efficient removal of ethanol. A higher fermentation temperature also increases the overall output of an ethanol plant. High ethanol tolerance is another important feature for the organism of choice for the development of SSF. In our laboratory, it was shown that expression of the $S$. cerevisiae MPR1 gene encoding $\mathrm{N}$-acetyltransferase, increases the ethanol tolerance of $H$. polymorpha (Ishchuk et al., 2010), thereby providing a useful approach for the construction of H. polymorpha strains with improved ethanol resistance. The heterologous expression of genes coding for amylolytic and xylanolytic enzymes, were successfully used in $H$. polymorpha, resulting in strains that are capable of direct starch and xylan fermentation at high temperatures (Voronovsky et al., 2009). In spite of the above mentioned achievements, the $H$. polymorpha yeast can only be considered for industrial ethanol production by further development of strains with a significant increase in its production of ethanol from xylose.

\section{Metabolic engineering of the initial stages of xylose catabolism}

For this we used several approaches. One line of research was directed to the substitution of the genes for xylose catabolism represented by the XYL1 gene coding for xylose reductase (XR) and two paralogs of xylitol dehydrogenase (XDH) XYL2A and XYL2B with the bacterial $x y l A$ gene from E. coli or Streptomyces coelicolor that code for xylose isomerase (XI) (Voronovsky et al., 2005). Such modification was used to avoid the cofactor imbalance arising from consecutive action of NADPH-dependent XR that reduces xylose to xylitol, and NAD-dependent XDH that oxidizes xylitol to xylulose. XI in turn directly converts xylose to xylulose with no cofactor requirement. The corresponding transformants that expressed XI activity grew on xylose, though the amount of accumulated ethanol was very low (up to $0.15 \mathrm{~g}$ of ethanol $\mathrm{L}^{-1}$ ). The overexpression of $E$. coli xylA together with $H$. polymorpha XYL3 coding for xylulokinase $(\mathrm{XK})$, led to increased ethanol production, but still the maximal ethanol accumulation did not exceed $0.6 \mathrm{~g} \mathrm{~L}^{-1}$ at $48^{\circ} \mathrm{C}$ (Dmytruk et al., 2008a). The second direction of research focused on the engineering of H. polymorpha XR with reduced affinity toward NADPH, using a similar approach developed earlier for Candida tenuis (Petschacher \& Nidetzky, 2005). Subsequently, genes coding for modified XR, native XDH and XK, were overexpressed in the CBS4732 strain which yielded a two-fold higher ethanol accumulation in corresponding transformants, reaching $1.3 \mathrm{~g}$ of ethanol L-1 (Dmytruk et al., 2008b). In a third line of research, the wild-type H. polymorpha strain NCYC495 was selected as the initial host, as it was shown to be a more efficient xylose fermenter, relative to the CBS4732 strain. The H. polymorpha mutant $2 \mathrm{EthOH}^{-}$unable to utilize ethanol as a sole carbon source was isolated from the NCYC495 strain and characterized by a 3-fold increase in ethanol accumulation. Subsequently, the PDC1 gene coding for pyruvate decarboxylase (PDC) was cloned and overexpressed in the $2 \mathrm{EthOH}^{-}$strain. The selected transformants derived from $2 \mathrm{EthOH}^{-}$, accumulated $2.5 \mathrm{~g}$ of ethanol $\mathrm{L}^{-1}$ at $48^{\circ} \mathrm{C}$ (Ishchuk et al., 2008).

In a more recent work (Kurylenko et al., 2014), we combined several approaches we developed earlier for the metabolic engineering with classical selection of 
ethanol overproducing strain, using selection for bromopyruvate resistance. We demonstrated that there is a positive cumulative effect for the overexpression of engineered XR and native $\mathrm{XDH}$ and $\mathrm{XK}$ on ethanol production from xylose in the background of the $2 \mathrm{EthOH}$ mutant. The additional overexpression of PDC did not lead to further improvement of ethanol synthesis from xylose, though the overexpression of PDC1 in the background of XYL1m and XYL2 overexpressing strain, increased ethanol production. The impact of XK on ethanol production during xylose alcoholic fermentation is more pronounced relative to PDC, assuming that PDC does not limit xylose conversion in a strain expressing $\mathrm{XR}, \mathrm{XDH}$ and XK. The overexpression of the XYL1m, XYL2 and XYL3 genes in the background of an unidentified mutation in the $2 \mathrm{EthOH}^{-}$strain, led to a substantial increase in ethanol accumulation during xylose fermentation $\left(7.44 \mathrm{~g} \mathrm{~L}^{-1}\right.$ at $45^{\circ} \mathrm{C}$, relative to $0.6 \mathrm{~g} \mathrm{~L}^{-1}$ in the wild-type NCYC495 strain and $2.05 \mathrm{~g} \mathrm{~L}^{-1}$ in the parental 2EthOH ${ }^{-}$strain) (Kurylenko et al., 2014). An additional increase in ethanol production from xylose (to $10 \mathrm{~g} \mathrm{~L}^{-1}$ at $45^{\circ} \mathrm{C}$ ), was obtained in mutants selected on a medium supplemented with toxic concentrations of 3-bromopyruvate, in a similar way to that described for ethanol accumulation from glucose in $S$. cerevisiae (Dmytruk et al., in press).

The maximum observed level of ethanol produced from xylose by the best strains isolated (nearly $10 \mathrm{~g} \mathrm{~L}^{-1}$ at $45^{\circ} \mathrm{C}$ ) represented an ethanol yield of $0.3 \mathrm{~g} \mathrm{~L}^{-1}$ from xylose. These results make $H$. polymorpha fall closely to the other known best xylose-fermenting organisms with relative ethanol production well-suited for the use in SSF process. Still, ethanol yield in $H$. polymorpha is lower than ethanol production reported for other mesophilic xylose fermenting organisms, such as P. stipitis (0.35-0.44 $\mathrm{g} \mathrm{g}^{-1}$ xylose) and $S$. passalidarum (0.42 $\mathrm{g} \mathrm{g}^{-1}$ xylose) (Jeffries et al., 2007; Long et al., 2012), but similar to the best engineered strain of the thermotolerant Kluveromyces marxianus yeast $\left(0.31 \mathrm{~g} \mathrm{~g} \mathrm{~g}^{-1}\right.$ xylose under anaerobic conditions at $45^{\circ} \mathrm{C}$ ) (Wang et al., 2013). However, the ethanol productivity in the best isolated strain of $H$. polymorpha is much higher than the best engineered strain of $K$. marxianus (0.179 $\mathrm{g} \mathrm{L}^{-1} \mathrm{~h}^{-1}$ versus $0.054 \mathrm{~g} \mathrm{~L}^{-1} \mathrm{~h}^{-1}$ at $45^{\circ} \mathrm{C}$ ) (Kurylenko et al., 2014). To be industrially feasible, ethanol yield in $H$. polymorpha has to be further increased to be close to the theoretical maximum.

\section{Autophagy-related gene ATG13 is important for xylose fermentation}

The 3-bromopyruvate appears to be a useful antimetabolite for selection of $H$. polymorpha strains with increased performance of xylose alcoholic fermentation (Kurylenko et al., 2014). Insertional mutagenesis was used to shed light on the nature of ethanol producing mutants of $H$. polymorpha that are resistant to 3-bromopyruvate. Cassette pL2 (Dmytruk et al., 2006) was used for transformation of NCYC495 strain of $H$. polymorpha, with subsequent selection of transformants on a mineral medium supplemented with $25 \mathrm{mM}$ 3-bromopyruvate. One transformant, among others selected, revealed a reproducible increase in ethanol accumulation during xylose fermentation. This transformant (\#63) had a $40 \%$ increase in ethanol production as compared to the parental strain, reaching $1.5 \mathrm{~g}$ of ethanol $\mathrm{L}^{-1}$ (unpublished data). We were able to isolate an insertion cassette with flanking regions from the strain. Sequencing of the flanking regions indicated that the insertional cassette disrupted the ORF of a gene homologous to the $S$. cere- visiae ATG13 gene encoding a regulatory subunit of the Atg1 signaling complex, stimulating Atg1 kinase activity, which is required for vesicle formation during autophagy and the cytoplasm-to-vacuole targeting pathway. It was shown that the insertion cassette disrupts the ATG13 gene at the +1272 bp position from the initial ATG codon.

Confirmation that the observed increase in xylose alcoholic fermentation performance of strain \#63 is a result of insertion cassette integration, rather than a secondary mutation occurring elsewhere in the genome, is an essential part of the study. For construction of a deletion cassette, LEU2 gene of $S$. cerevisiae was flanked with noncoding regions of the ATG13 gene. The $\Delta$ atg13 strain of $H$. polymorpha was constructed by homologous recombination of the deletion cassette. The $\Delta$ atg13 strain produced an elevated amount of ethanol from xylose, similar to that of insertional strain. Our results let us to conclude that the autophagy-related ATG13 gene is somehow involved in regulation of xylose alcoholic fermentation in the $H$. polymorpha yeast. However, mechanism of such regulations remains to be elucidated. It is important to note that $\Delta$ atg 13 mutant showed defects in autophagic degradation of peroxisomal protein alcohol oxidase, whereas insertion mutant \#63 did not (unpublished data). Thus, the role of Atg13 protein in autophagy apparently differs from that in regulation of xylose fermentation.

\section{Peroxisomal transketolase (dihydroxyacetone synthase) and transketolase are important for xylose fermentation}

Further increase in metabolic flux from xylose to ethanol requires additional new approaches. All known microorganisms convert xylose to ethanol using the sequential action of the pentose phosphate pathway (PPP) and glycolysis. Xylose is first taken up by cell, and then is converted to xylulose-5-phosphate. In four reactions of the non-oxidative part of the PPP (ribulose-5-phosphate epimerase, ribose-5-phosphate isomerase, transketolase and transaldolase, encoded by RPE1, RKI1, TKL1 and TAL1 genes, respectively), xylulose-5-phosphate is converted to different compounds, including the glycolytic intermediates fructose-6-phosphate and glyceraldehyde-3-phosphate. The latter compound can be converted to ethanol. Our interest in PPP is due to the observation of severe decrease in xylose alcoholic fermentation by $H$. polymorpha strains with defects in peroxisome biogenesis (so-called pex strains) (unpublished data). In the pex strains, the peroxisomal enzymes are mislocalized to the cytosol which result in many cases in degradation and/or inactivation of these enzymes (Kiel et al., 2000). Among the proteins of the peroxisomal matrix, our attention was focused on peroxisomal enzymes potentially involved in xylose catabolism. An example of this is the peroxisomal transketolase enzyme, also known as dihydroxyacetone synthase Das1, that is involved in methanol metabolism (Waites et al., 1981). Another example is the peroxisomal transaldolase Tal2 enzyme with unknown functions.

To investigate the role of these enzymes in ethanol production during xylose fermentation, the corresponding DAS1 and TAL2 genes were overexpressed in H. polymorpha NCYC495 leu1-1 strain under control of strong constitutive promoter of GAP1 gene (encodes glycerol-3-phospate dehydrogenase) with the use of a plasmid for multicopy integration, pGLG61 (Sohn et al., 1999). The recombinant strains overexpressing DAS1 and TAL 2 revealed 4.6- and 1.5-fold increase in the spe- 
cific activity of the corresponding enzymes (unpublished data).

The performance of xylose alcoholic fermentation by constructed strains was studied. The overexpression of TAL2 gene resulted in a 1.5 -fold increase in ethanol production after four days of xylose fermentation, as compared to the wild-type strain. The effect of the overexpression of DAS1 gene was more pronounced. Strain overexpressing DAS1 gene synthesized 2.3-fold higher ethanol than that of the parental strain after four days of xylose fermentation (unpublished data). As a consequence, it was shown for the first time that derepression of the peroxisomal enzymes Das1 and Tal2, resulted in the activation of xylose alcoholic fermentation in $H$. polymorpha.

\section{Deletion of transcription activator CAT8 activates xylose alcoholic fermentation}

The CAT8 gene encodes a zinc-finger cluster protein that mediates derepression of a number of genes during diauxic shift, which is the transition between fermentative and nonfermentative metabolism (Hedges et al., 1995). Genomic studies have shown that at least 30 genes, encoding proteins involved in gluconeogenesis, ethanol utilization, and the glyoxylate cycle, are regulated by Cat8 (Haurie et al., 2001; Tachibana et al., 2005).

It was recently shown that the deletion of CAT8 gene coding for the carbon source-responsive transcriptional regulator in the Pichia guilliermondii yeast, resulted in the shift from glucose metabolism toward a fermentative metabolism at both, the metabolic and the transcriptional levels. The $\Delta$ cat 8 mutant had a 20 -fold increase in ethanol production, when compared to the wild type under aerobic fermentation of glucose (Qi et al., 2014).

A similar strategy was applied to the xylose fermenting yeast $H$. polymorpha to improve its fermentation capacity and to induce respiratory-fermentative metabolism. This, we believe, is due to a defect in the transcription of genes involved in gluconeogenesis that would redirect more xylose toward fermentation. The $\Delta$ cat 8 strains of $H$. polymorpha were constructed in the background of the wild-type strain. Gene conferring resistance to the nourseothricin antibiotic was flanked with noncoding regions of CAT8 gene. A deletion cassette was used for specific site integration via homologous recombination. The transformed strain had a defect in growth on the gluconeogenic substrates (glycerol, ethanol), whereas growth of this strain on glucose and xylose was not affected. The $\Delta$ cat 8 mutants did not show changes in ethanol production in glucose medium, whereas they accumulated up to 3-fold more ethanol in the medium with xylose (unpublished data).

These results led us to propose that the transcription regulator Cat 8 is involved in the repression of $x y-$ lose alcoholic fermentation and consequently its damage strongly activates this process. Such an approach could be useful for the construction of the industrial xylose fermenting strains with a knock out of the CAT8 gene to enable further improvement in ethanol production from xylose.

\section{CONCLUSIONS AND FUTURE PERSPECTIVES}

Production of 1 st generation bioethanol is the largest application of industrial yeast biotechnology. Further enhancement of its production is not possible to achieve due to increasing feedstock demand. However, it is possible to obtain visible elevation of 1 st generation etha- nol production via genetic manipulation of the $S$. cerevisiae strains, which would produce more ethanol due to reduction in the production of major byproducts, such as glycerol and especially that of biomass. It is possible to decrease biomass production by decreasing in intracellular ATP content, which in turn could be achieved by the activation of some ATP degrading enzymes or activation of a possible futile cycle dissipating ATP. We report here on construction of $S$. cerevisiae strains which have elevated activity of ATP degrading enzymes (vacuolar alkaline phosphatase $\mathrm{PHO} 8$ or bacterial apyrase $a p y)$ and activated two futile cycles, consisting either of phosphofructokinase and fructose-1,6-bisphosphatase, or pyruvate kinase and phosphoenolpyruvate carboxykinase. Ethanol production was increased most efficiently in recombinant strains overexpressing a native gene coding for alkaline phosphatase PHO8. We hope this approach could be successful for improvement of yeast strains used in real industrial ethanol production. Another successful and original approach developed by us for improvement of $S$. cerevisiae ethanol production from glucose is based on positive selection of ethanol overproducers as mutants resistant to several antimetabolites, of mostly glycolysis inhibitors. We suggest that utilization of both methods (classical selection for antimetabolite-resistant strains and subsequent cloning and overexpression in antimetabolite-resistant strains of PHOS gene) would provide very efficient ethanol producers with elevated yield and productivity in ethanol synthesis from glucose.

Real surplus in global ethanol production can be achieved with 2 nd generation ethanol from lignocellulosic feedstock. One key challenge is the lack of yeast strains, preferably thermotolerant, capable of efficient alcoholic fermentation of the major pentose sugar of lignocellulose, xylose, at elevated temperatures suitable for the process of simultaneous saccharification and fermentation. We work in this field with one of the most thermotolerant yeast species, $H$. polymorpha, which ferments xylose, glucose and cellobiose, however, yield in productivity of ethanol synthesis from xylose by the wild-type strains is extremely low (Ryabova et al., 2003; Dmytruk \& Sibirny, 2013). Using combination of approaches of metabolic engineering and classical selection, we isolated a strain of $H$. polymorpha, which accumulates 15-20 times more ethanol from xylose at $45^{\circ} \mathrm{C}$, relative to the wild-type strain (Kurylenko et al., 2014), however, yield and productivity in ethanol synthesis are still not high enough. In this review, we describe new, original approaches for the selection of improved ethanol producers from xylose. They include (i) knock out of the ATG13 gene involved in initiation of autophagy; (ii) derepression of $D A S 1$ coding for peroxisomal transketolase (also known as dihydroxyacetone synthase), and TAL2 coding for peroxisomal transaldoalse; (iii) knock out of the CAT8 gene coding for global transcriptional activator of genes involved in gluconeogenesis, respiration and utilization of alternative carbon substrates. It is important to underline that all three approaches specifically activate alcoholic fermentation of only xylose but not that of glucose. It is important to study whether the new approaches developed by us, will be successful in increasing the ethanol production from xylose by the advanced ethanol producer which we constructed (Kurylenko et al., 2014). If so, we hope to construct competitive producers of ethanol form xylose, regarding the ethanol yield and productivity, which will be able to grow and ferment under elevated temperatures $\left(45-48^{\circ} \mathrm{C}\right)$ suitable for simultaneous saccharification and fermentation. 
The possible role of peroxisomal enzymes of pentose phosphate pathway and of transcriptional activator Cat8 in xylose alcoholic fermentation was predictable; however involvement in this process of autophagy-initiating protein Atg13 is a mystery. Further studies are needed to elucidate the mechanisms of the identified protein's actions in xylose metabolism and alcoholic fermentation.

\section{Acknowledgements}

This work was supported in part by Polish grant of National Science Centre (NCN) DEC-2012/05/B/ NZ1/01657 awarded to AAS; Archer Daniels Midland Company (Decatur, IL, USA); and National Academy of Sciences of Ukraine (Grant Nos 5-15, 6-15 and 35-15).

\section{REFERENCES}

Bekesi JG, Molnar Z, Winzler RI (1969) Inhibitory effect of d-glucosamine and other sugar analogs on the viability and transplantability of ascites tumor cells, Cancer Res 29: 353-359.

Benisch F, Boles E (2014) The bacterial Entner-Doudoroff pathway does not replace glycolysis in Saccharomyces cerevisiae due to the lack of activity of iron-sulfur cluster enzyme 6-phosphogluconate dehydratase. J Biotechnol 171: 45-55. http.dx.doi.org/10.1016/j.jbiotec.2013.11.025.

Chambers PJ, Bellon JR, Schmidt SA, Varela C, Pretorius IS (2009) Non-genetic engineering approaches to isolating and generating novel yeast for industrial applications. In Yeast biotechnology: Diversity and applications. Kunze G, Satyanarayana T eds, pp 433-457. Springer Science+Business Media.

Dmytruk K, Kshanovska B, Abbas C, Sibirny A (2015) New methods for positive selection of yeast ethanol overproducing mutants. Bioethanol, in press.

Dmytruk KV, Semkiv MV, Sibirny AA (2012) Ethanol yield and reduction of biomass accumulation in the recombinant strain of Saccharomyces cerevisiae overexpressing ATPase. US Patent Application, 0088290:A1.

Dmytruk KV, Voronovsky AY, Sibirny AA (2006) Insertion mutagenesis of the yeast Candida famata (Debaryomyces hansenii) by random integration of linear DNA fragments. Curr Genet 50: 183-191. http://dx.doi.org/10.1007/s00294-006-0083-0.

Dmytruk OV, Dmytruk KV, Abbas CA, Voronovsky AY, Sibirny AA. (2008b ) Engineering of xylose reductase and overexpression of xylitol dehydrogenase and xylulokinase improves xylose alcoholic fermentation in the thermotolerant yeast Hansenula polymorpha. Microb Cell Fact 7: 21. http://dx.doi.org/10.1186/1475-2859-7-21.

Dmytruk OV, Voronovsky AY, Abbas CA, Dmytruk KV, Ishchuk OP, Sibirny AA (2008a) Overexpression of bacterial xylose isomerase and yeast host xylulokinase improves xylose alcoholic fermentation in the thermotolerant yeast Hansenula polymorpha. FEMS Yeast Res 8: 165-173. http://dx.doi.org/10.1111/j.1567-1364.2007.00289.x.

Doelle HW (1975) Bacterial metabolism, 2nd edition. New York: Academic Press.

Fernandez M, Gascon S, Schwencke J (1981) Some enzymatic properties of vacuolar alkaline phosphatase from yeast. Curr Microbiol 6 : 121-126.

Geschwind JF, Georgiades CS, Ko YH, Pedersen PL (2004) Recently elucidated energy catabolism pathways provide opportunities for novel treatments in hepatocellular carcinoma. Expert Rev Anticancer Ther 4: 449-457. http://dx.doi.org/10.1586/14737140.4.3.449.

Gombert AK, van Maris AJ (2015) Improving conversion yield of fermentable sugars into fuel ethanol in 1st generation yeast-based production processes. Curr Opin Biotechnol 33: 81-86. http.dx.doi. org/10.1016/j.copbio.2014.12.012.

Hahn-Hägerdal B, Galbe M, Gorwa-Grauslund MF, Lidén G, Zacchi $G$ (2006) Bio-ethanol — the fuel of tomorrow from the residues of today. Trends Biotechnol 24: 549-556. http://dx.doi.org/10.1016/j. tibtech.2006.10.004.

Handa M, Guidotti G (1996) Purification and cloning of a soluble ATPdiphosphohydrolase (apyrase) from potato tubers (Solanum tuberosum). Biochem Biophys Res Commun 218: 916-923. http://dx.doi. org/10.1006/bbrc.1996.0162.

Haurie V, Perrot M, Mini T, Jenö P, Sagliocco F, Boucherie H (2001) The transcriptional activator Cat8p provides a major contribution to the reprogramming of carbon metabolism during the diauxic shift in Sacharomyces cerevisiae. J Biol Chem 276: 76-85. http://dx.doi. org/10.1074/jbc.M008752200.

Hedges D, Proft M, Entian KD (1995) CAT8, a new zinc cluster-encoding gene necessary for derepression of gluconeogenic enzymes in the yeast Saccharomyces cerevisiae. Mol Cell Biol 15: 1915-1922.
Ishchuk OP, Abbas CA, Sibirny AA. (2010) Heterologous expression of Saccharomyces cerevisiae MPR1 gene confers tolerance to ethanol and L-azetidine-2-carboxylic acid in Hansenula polymorpha. I Ind Microbiol Biotechnol 37: 213-218. http://dx.doi.org/10.1007/s10295-0090674-0.

Ishchuk OP, Voronovsky AY, Abbas CA, Sibirny AA (2009) Construction of Hansenula polymorpha strains with improved thermotolerance. Biotechnol Bioeng 104: 911-919. http://dx.doi.org/10.1002/bit.22457.

Ishchuk OP, Voronovsky AY, Stasyk OV, Gayda GZ, Gonchar MV, Abbas CA, Sibirny AA (2008) Overexpression of pyruvate decarboxylase in the yeast Hansenula polymorpha results in increased ethanol yield in high-temperature fermentation of xylose. FEMS Yeast Res 8: 1164-1174. http://dx.doi.org/10.1111/j.1567-1364.2008.00429.x.

Jeffries TW, Grigoriev IV, Grimwood J, Laplaza JM, Aerts A, Salamov A, Schmutz J, Lindquist E, Dehal P, Shapiro H, Jin YS, Passoth V, Richardson PM (2007) Genome sequence of the lignocellulose-bioconverting and xylose-fermenting yeast Pichia stipitis. Nat Biotechnol 25: 319-326. http://dx.doi.org/10.1038/nbt1290.

Jensen P, Snoep J, Westerhoff H (2006) Method of improving the production of biomass or a desired product from a cell. US Patent, 20060094078:A1. 17.

Kaino T, Takagi H (2008) Gene expression profiles and intracellular contents of stress protectants in Saccharomyces cerevisiae under ethanol and sorbitol stresses. Appl Microbiol Biotechnol 79: 273-283. DOI 10.1007/s00253-008-1431-4.

Kaneko Y, Akio Toh E, Oshima Y (1982) Identification of the genetic locus for the structural gene and a new regulatory gene for the synthesis of repressible alkaline phosphatase in Saccharomyces cerevisiae. Mol Cell Biol 2: 127-137.

Kiel JA, Veenhuis M (2000) Peroxisomal matrix protein import. Suppression of protein import defects in Hansenula polymorpha pex mutants by overproduction of the PTS1 receptor Pex5p. Cell Biochem Biophys 32 Spring: 9-19

Klionsky DJ, Emr SD (1989) Membrane protein sorting: biosynthesis, transport and processing of yeast vacuolar alkaline phosphatase. EMBO J 8: 2241-2250.

Klionsky DJ, Herman PK, Emr SD (1990) The fungal vacuole: composition, function, and biogenesis. Microbiol Rev 54: 266-292.

Ko YH, Pedersen PL, Geschwind JF (2001) Glucose catabolism in the rabbit VX2 tumor model for liver cancer: characterization and targeting hexokinase. Cancer Lett 173: 83-91. http://dx.doi. org/10.1016/S0304-3835(01)00667-X.

Kurylenko OO, Ruchala J, Hryniv OB, Abbas CA, Dmytruk KV, Sibirny AA (2014) Metabolic engineering and classical selection of the methylotrophic thermotolerant yeast Hansenula polymorpha for improvement of high-temperature xylose alcoholic fermentation. $\mathrm{Mi}$ crob Cell Fact 13: 122. http://dx.doi.org/10.1186/s12934-014-01223.

Lippert K, Galinski EA, Trueper HG (1993) Biosynthesis and function of trehalose in Ectothiorbodospira halochloris. Antonie Van Leeuwenboek 63: 85-91.

Long TM, Su YK, Headman J, Higbee A, Willis LB, Jeffries TW (2012) Cofermentation of glucose, xylose and cellobiose by the beetle-associated yeast Spathaspora passalidarum. Appl Environ Microbiol 78: 5492-5500. http://dx.doi.org/10.1128/AEM.00374-12.

Navas MA, Gancedo JM (1996) The regulatory characteristics of yeast fructose-1,6-bisphosphatase confer only a small selective advantage. J Bacteriol. 178: 1809-1812.

Olofsson K, Bertilsson M, Lidén G (2008) A short review on SSF - an interesting process option for ethanol production from lignocellulosic feedstocks. Biotechnol Biofuels 1: 7. http.dx.doi.org/10.1186/17546834-1-7.

Panesar PS, Marwaha SS, Kennedy JF (2006). Zymomonas mobilis: an alternative ethanol producer. J Chem Technol Biotechnol 81: 623-635. http.dx.doi.org/10.1002/jctb.1448.

Petschacher B, Nidetzky B (2005) Engineering Candida tenuis xylose reductase for improved utilization of NADH: antagonistic effects of multiple side chain replacements and performance of site-directed mutants under simulated in vivo conditions. Appl Environ Microbiol 71: 6390-6393. http://dx.doi.org/10.1128/AEM.71.10.63906393.2005

Piškur J, Langkjaer RB (2004) Yeast genome sequencing: the power of comparative genomics. Mol Microbiol 53: 381-389. http.dx.doi. org/10.1111/j.1365-2958.2004.04182.x.

Plesner L (1995) Ecto-ATPases: identities and functions. Int Rev Cytol 158: 141-214. http://dx.doi.org/10.1016/s0074-7696(08)62487-0.

Qi K, Zhong JJ, Xia XX (2014) Triggering respirofermentative metabolism in the crabtree-negative yeast Pichia guilliermondii by disrupting the CAT8 gene. Appl Environ Microbiol 80: 3879-3887. http:// dx.doi.org/:10.1128/AEM.00854-14.

Rogers DT, Szostak JW (1993) Strains of yeast with increased rates of glycolysis. US Patent, 5268285:A1. 16.

Ryabova OB, Chmil OM, Sibirny AA (2003) Xylose and cellobiose fermentation to ethanol by the thermotolerant methylotrophic yeast Hansenula polymorpha. FEMS Yeast Res 4: 157-164. http://dx.doi. org/10.1016/S1567-1356(03)00146-6. 
Semkiv MV, Dmytruk KV, Abbas CA, Sibirny AA (2014) Increased ethanol accumulation from glucose via reduction of ATP level in a recombinant strain of Saccharomyces cerevisiae overexpressing alkaline phosphatase. BMC Biotechnol 14: 42. http.dx.doi.org/10.1186/14726750-14-42.

Sohn J, Choi E, Kang H, Rhee J, Agaphonov M, Ter-Avanesyan M, Rhee S (1999) A dominant selection system designed for copy-number-controlled gene integration in Hansenula polymorpha DL-1. Appl Microbiol Biotechnol 51: 800-807.

Tachibana C, Yoo JY, Tagne JB, Kacherovsky N, Lee TI, Young ET (2005) Combined global localization analysis and transcriptome data identify genes that are directly coregulated by Adr1 and Cat8. Mol Cell Biol 25: 2138-2146. http://dx.doi.org/10.1128/MCB.25.6.21382146.2005.

Taxis C, Knop M (2006) System of centromeric, episomal, and integrative vectors based on drug resistance markers for Saccharomyces cerevisiae. Biotechniques 40: 73-78. http://dx.doi.org/10.2144/000112040.

Tylicki A, Czerniecki J, Dobrzyn P, Matanowska A, Olechno A, Strumilo S (2005) Modification of thiamine pyrophosphate dependent enzyme activity by oxythiamine in Saccharomyces cerevisiae cells. Can I Microbiol 51: 833-839. http://dx.doi.org/10.1139/W05-072.

Uhlemann H, Schellenberger A (1976) Glyoxylic acid as an active site marker of yeast pyruvate decarboxylase. FEBS Lett 63: 37-39. http://dx.doi.org/10.1016/0014-5793(76)80189-5.

Voronovsky AY, Ryabova OB, Verba OV, Ishchuk OP, Dmytruk KV, Sibirny AA (2005) Expression of $x y l A$ genes encoding xylose isomerases from Escherichia coli and Streptomyces coelicolor in the methylotrophic yeast Hansenula polymorpha. FEMS Yeast Res 5: 1055-1062. http://dx.doi.org/10.1016/j.femsyr.2005.09.001.

Voronovsky AY, Rohulya OV, Abbas CA, Sibirny AA (2009) Development of strains of the thermotolerant yeast Hansenula polymorpha capable of alcoholic fermentation of starch and xylan. Metab Eng 11: 234-242. http://dx.doi.org/10.1016/j.ymben.2009.04.001.

Wang R, Li L, Zhang B, Gao X, Wang D, Hong J (2013) Improved xylose fermentation of Kluyveromyces marxianus at elevated temperature through construction of a xylose isomerase pathway. J Ind Microbiol Biotechnol 40: 841-854. http://dx.doi.org/10.1007/s10295013-1282-6. 